\title{
EXCURSION A HUÉ, CAPITALE DE L'ANNAM.
}

\author{
PAR \\ CH. LEMIRE.
}

On peut se rendre à Hué en partant de Tourane par la route mandarine. La distance est de 105 kilomètres, divisés en 10 étapes. On traverse le village de Gia lé, puis celui de Tham lam après avoir passé en bac le Song (fleuve) Vuc où s'étale uu grand marché, entouré d'arbres à fruits. La résidence du huyen de Phu loc est sur la route. On franchit ensuite un plateau de sable de 5 kilom. au bout duquel est le tram de Thua nong, où l'on trouve les relais de porteurs et les hommes de la poste. Ceux-ci reçoivent par mois 30 rations de riz et un frane de solde. C'est maigre pour un service quotidien de jour et de nuit. Chaque tram a pour chef un doi. C'est une sorte de caravansérail pour les fonctionuaires en voyage. Les trams ont été créés en Annam par le roi Ly Thai Tong de 1042 à 104.7. C'est ì dire bien des siècles avant la poste française. Ces stations postales servaient aussi de logement aux voyageurs et aux ambassadeurs dı Cambodge et du Ciampa (Kiams).

Le nom d'un tram est toujours formé d'un des deux mots formant le nom de la province. Ainsi dans la province de Quang Duc où est située Hué qui s'appelle plus souvent Thu'a-tiên, les trams se nomment Thu'a nong, Thu'a hoa, etc.

On nomme en même temps tram la maison de poste et les courriers à pied ou à cheval, qui courent en faisant résonner un grelot attaché ì leur poignet.

Le second tram est celui de Thu'a hoa sur le Song Nong, qu'on passe en bac pour arriver à Câu hai.

C'est l'extrémité d'une grande lagune qui commnnique avec Thuân-an et avec Hué par la rivière de Phu cam. On trouve toujours à Câu hai et à Trûoi des barques pour se rendre de là à Hué par eau. En barque la distance de Câu hai à Trûoi 
est de 2 heures. En 3 heures on va de Câu hai à Trûoi par une route bordée de beaux arbres. On y trouve un marché bien approvisionné. La route est coupée par le col de Câu doi. On pense qu'avant 1958 un canal mettait en communication Câu hai avec le Song Cudé qui se jette daus la baie de la Tourane. Nous en parlons plus loin.

Les vapeurs font le trajet de Tourane à Hué en 8 heures. Ils franchissent la barre de Thuân-an et remontent la rivière qui communique avec la lagune de Tru vi. Thuân-an (ou Dai-an) est a 14 kil. de Hué. Il y existe un sémaphore, une ambulance, un poste militaire, un régiment européen, une mission catholique, un bureau de télégraphe et de poste. Les chaloupes à vapeur de et pour Hué, les nombreuses barques, les pêcheries, donnent quelque animation à ce port intérieur. Les dunes sont plantées de quelques palmiers sur la rive droite, autour des anciens forts et dans l'île Hai do. On atteint bientôt le village de Ba truc. Les rives deviennent verdoyantes. De ravissantes pagodes se dessinent sous les gros banians, jusqu'au croisement de la rivière et du canal devant le Mang-ca. On nomme ainsi la partie de la citadelle réservée à nos troupes et à divers services européens. En général, les vapeurs mouillent à Dong ba. On prend là un sampan et l'on traverse la ville établie sur les deux rives du canal. Le parcours est très pittoresque et très animé. Des pagodes avec portiques, encadrées d'ombrages, se succèdent et alternent avec les boutiques des annamites et des chinois.

Une scierie à vapeur, une usine à glace, une fabrique d'eaux gazeuses et un magasin installés par des français, les frères Bogaërt, sont situés à l'entrée du canal.

Des quais ont été construits. Des ponts ont été créés ou rendus praticables aux voitures attelées.

La Légation est sur la rive droite. C'est un grand bâtiment à étage, avec dôme central, d'un aspect imposant. Il est a $700 \mathrm{~m}$. des remparts de la citadelle.

Les casernements des troupes sur la rive droite sont en bordure de la route de Tourane.

Les postes et télégraphes sont à gauche de la Légation.

Si l'on suit la rive gauche du fleuve on longe la citadelle, on passe devant le village de Kim long où sont établis l'évêché, la mission, la $\mathrm{S}^{\text {te }}$ Enfance, l'habitation de l'ancien Kinh Luoc du Tonkin, Nguyen Húu Do, et l'on arrive à l'angle nordouest du 
canal. Cette pointe est occupée par des pagodes Boudhiques et par le temple de Confucius.

Deux édicules flanquent une tour de style chinois, à 7 étages, en avant de laquelle un péristyle et des escaliers précédés de pilastres conduisent du fleuve à la tour. L'ensemble de ces monuments étagés forme un joli paysage. Des bosquets touffus renfermant des temples se succèdent sur la rive jusqu'à un promontoire très boisé, où l'on aperçoit dans le feuillage les toits ouvragés et dentelés de la pagode élevée par Dong Khanh à sa nourrice. Il faut prendre un sampan pour aller en 6 heures jusqu'au tombeau de Minh Mang et en 8 heures ì celui de Gia Long, tous deux sur la rive gauche; mais sur deux bras différents de la rivière.

Pour s'y rendre par la rive droite, on peut aller à cheval de Hué jusqu'ì un affluent où l'on prend un sampan qui mène en une demi-heure au débarcadère de Minh Mang. Le sentier passe ì travers les nécropoles de Dong Khanh, Tu Due et Thieû 'Tri.

On va aussi de Hué directement en sampan aux débareadères de Tu Duc et de Thieû Tri sur la rive droite.

Les voitures peuvent aller de Hué (rive droite) par le pont de Phu Cam jusqu'au delà de l'Esplanade des sacrifices.

Nous partons à cheval. Nous traversons la rivière de Phu Cam et le marché. Nous laissons ì droite les arènes royales abandonnées, où avaient lieu autrefois des combats d'animaux. Près de là on retrouve les traces de l'enceinte d'une très ancienne citadelle des Kiams ou Tsiampois.

A gauche, une montagne en forme de trapèze dont les crêtes sont garnies d'une dentelure de pins sert d'écran naturel au Palais; de là son nom de montagne ou d'écran du roi.

Voici la grande enceinte carrée à 4 portes renferınant des quinconces de pins séculaires. Ils ont été plantés par les membres des familles royales et chaque arbre porte sur une tablette en métal le nom de celui qui l'a planté.

Deux terrains carrées superposées, à balustrades ajourées, entourent une troisième terrasse ronde, au centre de l'esplanade.

C'est l'aire des grands sacrifices.

Sur les quatre côtés s'ouvrent des escaliers orientés selon les quatre points cardinaux.

Lorsqu'au milieu de ces allées de pins, le cortège royal gravit dans la nuit ces escaliers éclairés par des torches, c'est un spec- 
tacle imposant. Les mandarins en grand costume rangés sur les marches, les tables d'offrandes, les pavillons, les insignes royaux, les grands brûle-parfums, les orchestres, les gongs et tamtans, les mélopées des maîtres de cérémonies, tout cet appareil donnent ì la cérémonie du Nam Giao un caractère solennel et grandiose ${ }^{1}$.

Dans une autre partie de l'enceinte s'élèvent plusieurs édifices clos de hautes murailles. C'est, là que le roi, les ministres, les princes se retirent pour observer la retraite, le jeûne et l'abstinence qui précèdent l'offrande des grands sacrifices.

Sur la droite, on gravit une colline dont la crête est couronnée de pins. De ce point on jouit d'un splendide panorama, embrassant les vallées d'où émergent les monuments des nécropoles royales, les tombeaux en pyramides, les pylônes enfouis dans la verdure.

On parvient bientôt a la nécropole de Dong Khanh, le dernier roi. La durée du règne (de 14. Septembre 1885 au 28 Janvier 1889) a été trop courte pour achever l'oeuvre à laquelle les souverains président de leur vivant. Ils en sont à la fois les architectes, les régisseurs et les occupants. Par leur ordres et sous leur direction, une armée de plusieurs milliers de travailleurs, coolies, maçons, peintres, charpentiers, forgerons, fondeurs, jardiniers, briquetiers, carriers, sculpteurs, incrusteurs, se rendent sur l'emplacement choisi par le royal constructeur. Pendant de longues années, on approprie les accideuts du sol, on dalle des avenues, des cours, des terrasses, on plante des arbres, des bosquets, des jardins; on creuse des bassins, on les alimente d'eau et on assure l'écoulement des eaux de pluie. On dresse des enceintes de murailles, on élève les bâtiments, les portiques, on construit les ponts, on prépare le caveau.

L'ensemble d'une nécropole comprend une entrée monumentale à trois ouvertures cintrées à triple dôme, conduisant à une large cour dallée analogue à l'entrée du palais de Versailles. De chaque côté se dressent en grandeur naturelle les statues des ministres et grands mandarins, les civils d'abord, la règle d'ivoire à la main, comme les anciens sénateurs de Rome; puis les militaires, l'épée au côté; puis les deux chevaux du roi, ses deux éléphants de guerre, taillés d'un seul bloc. Sur leur piédestal, les deux grands dragons dorés à 3 griffes, symbole de la dynastie, gardent la première terrasse. Trois grands escaliers, celui du milieu étant réservé au roi,

1 Voir l'étude sur „le Culte officiel des annamites", par Ch. Lemire (1893). 
mènent ì la seconde et à la troisième terrasse. Au milieu s'élève le dôme ì étage qui abrite la stèle royale. C'est un monolithe énorme en grès poli qui repose sur un soubassement sculpté. Ces blocs out 3 mètres de large sur 4 mètres de haut. On les apporte généralement des carrières du Thanh-hoa, e'est à dire de 350 kilomètres; et le travail de sculpture, de gravure et de transport exige plusieurs années. C'est ce qui explique comment le roi défunt Dong Khanh n'a pas encore de stèle, tandis que son père en a une dressée par les soins de son royal fils.

Les européens croient à première vue que ces épitaphes recouvrent la tombe des rois. Il n'en est rien; le tombeau forme une enceinte à part dans la partie la plus reculée de la nécropole, et se reconnaît aux portiques impériaux qui précèdent les portes de bronze toujours fermées, et qui ne s'ouvrent qu'une fois l'an devant le roi seul. ('est la troisième partie de la nécropole. On n'y entre jamais. On n'en voit que la porte.

En 1892, le Gouverneur Général, et le Résident Supérieur M. Brière, furent admis pour la première fois à pénétrer avec le jeune roi Thanh Thai, agé de 14 ans, de la dynastie des Nguyen, dans l'enceinte où repose son illustre aïeul Gia Long (mort en 1819), et à contempler son tombeau.

Un grand brûle-parfums en bronze à anses et à quatre pieds est placé devant la stèle.

Le dôme de la stèle est naturellement la première partie de la nécropole. La seconde partie est la pagode mortuaire, la nha-tu, la chapelle funèbre qui est véritablement l'habitation des mânes du défunt.

Des stores peints et des étoffes jaune et rouge en garnissent les entrées; on y pénètre par 3 portes dorées, ornées de dragons. Une perpétuelle obscurité règne dans l'intérieur et l'on ne s'y dirige qu'à l'aide d'une lumière.

$\mathrm{Ce}$ palais se compose de plusieurs salles soutenus par des colonnes, laquées rouge et or comme les boiseries, les chevrons sculptés, les tables etc. Le sol est en carreaux vernissés. Au milieu est suspendu un lustre à cristaux de fabrication européenne, et des lanternes à verres peints. Sur des guéridons devant chaque colonne un cadre de verre abrite une corbeille, soit en émail de Hué, soit en cristal de roche, soit en lames d'or, contenant les fleurs d'or que les peuples tributaires apportaient autrefois comme hommage. Des vases européens en porcelaine décorée avec de fausses fleurs, sont également placés sous globes. Au-dessus de chaque 
pilier, dans des cadres dorés, des tableaux chinois à cadre doré représentent les attributs traditionnels annamites: l'épée, le brûleparfums, l'aiguière à vin de riz, les coffrets, les pinceaux, etc.

Sur les côtés on remarque des vases de bronze très anciens, de formes bizarres et ornés de caractères en saillie.

$\mathrm{Au}$ milieu et au fond de la pièce s'ouvrent les portes dorées et ajourées d'un autel laraire. Le lit royal entouré de tentures jaunes se dresse en avant. Au centre de la première travée l'autel ordinaire est garni de brûle-parfums, de vases de fleurs, et de grands chandeliers.

A droite, la table de toilette supporte les aiguières et les bassins; sur des séchoirs les linges sont prêts à servir. Du vestiaire pendent les robes et vêtements royaux, et au-dessous s'alignent les souliers chinois en soie brodée d'or et à grosse semelle de feutre blanc.

Sur la table (phan) à manger, avec les supports des plats, les deux grands vases en porcelaine bleue pour l'eau et le vin, tout est préparé pour un repas.

A gauche sont conservés les ustensiles pour écrire, les cachets en jade, les sceptres, les boîtes incrustées, ou en ivoire à jour, armées de pierreries, ou en argent repoussé ou filigrané, les boîtes à bétel que l'on garnit de chiques toutes préparées et renouvelées. On y a rassemblé les ustensiles qui rappellent l'existence et les fonctions royales. 1

Dans ces habitations posthumes, au milieu des trésors royaux, à la lueur vacillante d'une pauvre veilleuse, les femmes du défunt et ses anciens serviteurs veillent à perpétuité. De sorte que l'ombre du souverain $y$ retrouve tous les ustensiles à son usage, tout le nécessaire de la vie et son entourage ordinaire, prêt à le servir.

Dans la nécropole de Minh Mang vit une vieille femme de 78 ans qui y est cloîtrée depuis 1840 , date de la mort de son auguste époux. Elle aime à recevoir elle-même les visiteurs qui laissent généralement une offrande à ses servantes. Après avoir salué cette veuve royale, les gardiens et gardiennes de la pagode conduisent les étrangers.

Dans les magasins latéraux sont les parasols, les malles royales laquées noir et incrustées de nacre, les paniers à provisions de

1 Il est à remarquer que dans ce mobilier royal, on ne trouve aucun sabre, aucune épée, aucune arme, bien que ce soit un attribut royal. On se rappelle que le sabre de Gia Long, orné de diamants, fut confié par un officier au général De Courcy et envoyé en France. On aimerait à savolr où l'on a placé cette pièce de grande valeur intrinsèque et surtout historique.

6e Volgr. VI. 
bouche, en laque rouge dorée, les palanquins aux stores jaunes, les tables incrustées, les lanternes peintes, les lances, hallebardes, masses, et autres insignes. Dans le magasin de Minh Mang est un guéridon superbe, présent de Louis XVI au roi Gia Long.

Derrière la grande pagode s'étagent des parterres entourés de balustrades en tuiles ajourées, moulées et vernissées. Tout autour sont placés sur des piédestaux en pierre de grands vases a fleurs de fabrication chinoise et de la famille bleue.

Les portiques qui précèdent et terminent les ponts sur les bassins sont formés de quatre colonnes de bronze, autour desquelles s'enroule le dragon impérial. Elles sont surmontées de fleurs de lotus et rattachées les unes aux autres par des plaques de porcelaine à fleurs peintes et à fond alternativement bleu et jaune.

Les rampes d'escalier figurent des dragons en pierre, la perle dans la gueule, et dont les replis écaillés se déroulent de degré en degré.

La nécropole la plus proche est celle de Dong Khanh. Les femmes guident le visiteur dans la pagode; mais on ne pénètre pas dans le fond qui est sur un soubassement plus élevé. Les ouvertures en sont garnies de verres de couleur.

Dans le compartiment voisin, habitent le jeune fils du roi et ses autres enfants. Un vieux professeur leur apprend les caractères. Le jeune prince portait, quand nous l'avons vu, une robe de brocard damassée rouge et or. Les petits doigts étaient chargés de bijoux. Sa physionomie était tout a fait avenante.

Cette nécropole n'a pas d'enceinte; ses jardins ne sont qu'ébauchés. Les bassins ne sont que des rizières.

Un peu plus loin, on reconnaît le tombeau du père de Dong Khanh aux deux édicules qui le flanquent. Les sujets en relief peints sur les faces représentent des scènes de piété familiale: le fils qui sauve son père de l'attaque du tigre; la fille qui nourrit de son lait son vieux père.

En suivant le sentier qui descend de la colline, on arrive á la porte de la nécropole de Tu Duc, qui règna de 1847 à 1883. Elle est en tuiles ajourées et à clochetons superposés, dont les angles sont garuis de clochettes que le vent fait résonner.

On suit une allée dallée qui longe les bassins, à droite. Une île y répand sa verdure. De vastes escaliers conduisent à la grande pagode d'où l'on domine. un coquet paysage. Les toitures en pointe, les portiques, émergent des bosquets. En bas, un petit pavillon 
précède l'escalier accédant au bassin, et à gauche, ì fleur d'eau, une pittoresque maison de bains borde le lac au fond duquel deux pirogues sont amarrées.

La forme tourmentée des bassins, l'entre-croisement des balustrades et des ponts, les arbustes qui en garnissent le tour et les bosquets qui les surplombent, rendent ce séjour plus attrayant que funèbré.

Dans la pagode, le trésor royal est très curieux. Il y a des jades, des émaux, des ivoires, des cristaux, des objets de grand prix. Tu Duc n'étant mort qu'en juillet 1883 après un règne de 36 ans, sa dernière demeure a été achevée soigneusement. Les laques et les ors sont d'uue grande fraîcheur.

Pour visiter la pagode, il est nécessaire de le demander aux gardiens en déclinant ses qualités.

La stèle royale se trouve dressée entre quatre colonnes sous un dôme imposant au milieu d'une terrasse qui est i droite de la pagode et en face du tombeau. Deux grands pylônes ornés de tuiles ouvragées flanquent le monument, qu'entourent des frangipaniers, des litchis, des hibiscus, etc. Des dragons aux vives écailles courent sur les crêtes des toitures ou sont accroupis aux angles, la gueule béante et les yeux menaçants. Leur corps est formé de tessons de porcelaines. En voyant cette profusion inouie de morceaux de vases dans les décorations, les soubassements, les piliers, les balustrades et jusque sur les crêtes des murs d'enceinte, on se demande combien de millions de tasses chinoises il a fallu rassembler et briser pour faire emploi de tant de morceaux miroitants et cimentés sur toutes les murailles et les toitures!

Dans plusieurs pagodes des nécropoles, on voit encadrées de mauvaises lithographies de fleurs servant à l'étude du dessin et qui détonent au milieu de ces trésors de l'orient.

Dans la même enceinte et sur la gauche du tombeau de Tu Duc s'élèvent en amphithéâtre les bâtiments servant de tombeau à Kien Phu'oc. Ce petit roi était tout jeune lorsqu'il monta sur le trône. Il ne règna que 7 mois. Son intronisation n'ayant pas été ratifiée par le Protectorat, les régents l'empoisonnèrent le 31 Juillet 1884, comme ils avaient fait mourir violemment Duc Du'c et Hiep hoa le 30 Décembre 1883, pour avoir au lendemain de la prise de Hué accordé aux représentants de la France la première audience directe et personelle.

Dans la pagode royale de Kien Phu'oc, les femmes éclairent de la lueur blafarde d'un cierge les jouets, les oiseaux chanteurs 
et désormais muets, les cadeaux que le jeune prince avait reçus de la France. Quel contraste entre ce luxe enfantin et cette fin si tragiquement prématurée!

Poursuivant la route a travers les larges avenues de jacquiers, de pins, de cay mu'u, on aperçoit bientôt au-dessus de la cime des arbres le lotus sacré qui couronne les énormes pylônes entourant la stèle de Thieû Tri, fils de Minh Mang (1841 à 1847).

La nécropole est divisée en deux enceintes juxtaposées. Dans l'une la pagode aux portes dorées, dont les escaliers dominent un vaste bassin. Dans l'autre la vaste cour où sont rangés les mandarins de pierre, puis la terrasse au milieu de laquelle s'élève le dôme qui abrite la stèle. Derrière sont les jardins. Puis les portiques s'ouvrant sur les ponts jetés sur les pièces d'eau et au fond l'enceinte du mausolée et au milieu ses portes de bronze cadenassées et verrouillées, entourées de plantations de pins et de superbes bosquets en amphithéâtre.

L'aspect de cette nécropole est plus imposant, et son caractère plus antique revêt une religieuse solennité.

En la quittant, pour suivre le sentier qui longe la rive droite du fleuve on traverse un sombre bois de sapin où s'élève dans l'ombre une plus modeste nécropole. C'est celle du père de Gia Long.

Plus loin, on abandonne les chevaux et un sampan nous conduit sur la rive droite à l'entrée de l'avenue de Minh Mang (1820 a 1840).

Nous pénétrons avec les gardiens dans l'enceinte. La perspective en est admirable, au delà du vaste bassin qui entoure les bâtiments. On le traverse a gauche sur un pont rustique et l'on a devant soi la cour peuplée de mandarins en effigie et l'ensemble des bâtiments qui se succèdent en droite ligne, au milieu des terrasses superposées et bordées de vieux arbres. Ces bâtiments sont séparés par des jardins et des bassins du mausolée royal adossé à la montagne boisée. L'ensemble est harmonieux et grandiose.

Le sampan nous ramène sur la branche principale du fleuve et l'on accoste à l'avenue de Gia Long. Les avenues de Tu Duc et de Thieû Tri sont les seules qui soient munies de débarcadères en pierres et en bois. L'avenue de Minh Mang n'a pas plus de 300 mètres de longueur. Celle de Tu Duc et de Thieu Tri ont 1 kilomêtre et celle de Gia Long 2 kilomètres et demi. Celles de Minh Mang et de Gia Long, la dernière surtout, sont très peu praticables en 
hiver ou en temps de pluie. En outre, les chemins dallés et les escaliers de marbre sont très glissants et dangereux dans la même saison.

On laisse ì droite le modeste mausolée du prince Canh, fils de Gia Long. Ce monument dans sa simplicité rappelle les souvenirs du voyage du jeune prince en France et des espérances que son séjour à Versailles, il y a juste un siècle, avait fait concevoir. Sa fin prématurée, à 22 ans, fut une grande perte pour l'influence française qui devait aller en s'amoindrissant après la mort de Gia Long.

Plus loin, à droite, s'étend la nécropole de la mère de ce souverain.

On entre dans la nécropole du roi par une porte opposée aux façades des bâtiments. Le mausolée et la pagode royale en effet se font vis-à-vis, et c'est vers le tombeau que descendent les grands escaliers, les terrasses, et les jardins, qui en sont séparés par des bassins et des ponts.

Dans la pagode, on ne retrouve plus que le lit de repos du grand roi. Les portes et les architraves sont massives et sculptées en bosse; mais la laque est ternie; les ors ont disparu; aucun objet de prix ne subsiste à l'intérieur. Les bâtiments et leurs alentours sont mal entretenus.

Ce qui donne à cette nécropole un cachet special, e'est la sauvagerie imposante du site, un enchevêtrement de collines boisées, de ravins, de jardins étagés, de bassins profonds, de hauts et rapides escaliers, que relient des dragons de pierre noircis par le temps. On remarque, çà et lì, des arbres à pain que les annamites appellent $\mathrm{Xa}$ Ké (artocarpus incisafolia). De grands banians, des pius séculaires, des euphorbes aux tiges serpentueuses, des litchis couverts de mousse, une nature abrupte et accidentée, la décrépitude des bâtiments, tout indique que nous reculons dans un passé plus éloigné. Les dynasties ont varié. Les princes du sang out disparu. Les courtisans sont morts. Les épouses qui veillaient à l'entretien des bâtiments ont rejoint leur royal maître.

Il y a comme un indice d'abandon auquel le jeune roi, en qui revit la dynastie des Nguyen, a déjà pensé à remédier. La mémoire du grand roi auquel l'Annam doit ses citadelles, ses routes, ses monuments, ses grands travaux et une partie de ses grandes institutions, mérite que son mausoleé soit mis a l'abri des injures du temps.

Lorsqu'en mai 1892 le jeune roi Thanh Thäi est allé rendre ses hommages avec toute sa cour et en présence des représentants de 
la France à son illustre ancètre, quelles pensées out dû assaillir son jeune esprit? Tant d'oeuvres grandioses ont été, au milieu des plus grandes difficultés, d'incessantes péripéties, de luttes opiniâtres, accomplies par son prédécesseur, que la tâche doit l'effrayer. Il m'a été donné de le voir (en 1891) assis sur son trône, ses petits pieds reposant sur la tête des dragons dorés. Sa physionomie était grave et soucieuse, intelligente et énergique. Puisse son règne être encore plus long que ceux de Tu Duc et de Ly Nho'n Tong!

L'impression qui ressort de la visite des nécropoles royales est une révélation de la grandeur et de la vitalité du royaume, dans un passé encore bien près de nous. La majesté royale s'y affirme dans le silence funèbre, comme dans la vie. A la cour d'Annam on ne parle qu’à voix basse. Les paroles du roi sont à peine perceptibles. Les ministres errent dans ces vieux palais comme des ombres muettes dans leurs longs vêtements bleus.

Même à la lueur des flambeaux, la lumière y pénètre à peine. Le séjour des rois vivants est moins luxueux et moins agréable que celui des rois défunts.

Chaque nécropole est un Trianon où règne un éternel printemps, et où les bosquets gardent toute l'année leur verdure et leurs fleurs. Voici des monuments pittoresques, au milieu d'une nature luxuriante embellie par l'art.

Dans ses traditions et ses moeurs nationales, comme dans son culte funéraire pour ses souverains, le royaume d'Annam a su honorer et perpétuer leur mémoire d'une façon beaucoup plus solennelle, plus charmante, et plus imposante à la fois, que nous n'avons su le faire pour nos morts les plus illustres. Ce n'est pas la seule leçon de haute philosophie et de grandeur de sentiments que le protégé ait offertıau Protecteur.

Hué, le 24. Décembre 1894.

Ch. Lemire. 